Check for updates

Cite this: J. Anal. At. Spectrom., 2022 37,883

Received 23rd November 2021 Accepted 21st February 2022

DOI: $10.1039 / \mathrm{d} 1 \mathrm{ja} 00402 \mathrm{f}$

rsc.li/jaas

\title{
Line plasma versus point plasma VUV LIBS for the detection of carbon in steel: a comparative study
}

\author{
Syedah Sadaf Zehra, (D)*ac Lazaros Varvarezos, (D) a Paddy Hayden, \\ Piergiorgio Nicolosi, ${ }^{\mathrm{c}}$ Paola Zupella, ${ }^{\mathrm{d}}$ Muhammad B. Allia and John Costello ${ }^{\mathrm{a}}$
}

\begin{abstract}
Steel impacts a variety of industries such as construction, automotive and ship building. The physical properties of steel (e.g., hardness, weldability) are significantly affected by the concentration of carbon. Hence, accurate determination of carbon content in steel has been, and still is, an active field of research. Laser induced breakdown spectroscopy (LIBS), has been extensively used applied to measure the concentration of carbon in steel. Specifically, vacuum-ultraviolet (VUV) LIBS is a favourable technique for detection of light elements. In this work, a line plasma, as opposed to the more commonly used point like plasma plume, is explored to systematically investigate its effect on the limit of detection for carbon in steel. In addition, the effect of moving the target surface and cylindrical lens focus with respect the optic axis of the spectrometer is studied. The presence of a fore slit, between the plasma and entrance slit of the spectrometer, provides a degree of space resolution, by allowing radiation from a specific slice of the plasma plume to enter the spectrometer. Importantly, the experimental findings suggest a significant improvement in the relative limit of detection, by a factor of six, over the more usual point-like plasmas.
\end{abstract}

\section{Introduction}

Laser-induced breakdown spectroscopy (LIBS) is a robust analytical technique that uses atomic ${ }^{1,2}$ and/or molecular ${ }^{3}$ emission spectrometry of Laser Produced Plasmas (LPPs) to determine the elemental composition of solids, liquids or gases. Several advantages are accredited to LIBS including, but not limited to: little or no sample preparation, fast data acquisition and ability to detect any chemical element in complex hosts or matrices. ${ }^{4,5}$ In light of such compelling advantages, LIBS has found application in a broad range of fields (e.g. detection of explosives, ${ }^{6-8}$ preservation of culutral heritage, ${ }^{9-14}$ metallurgy, ${ }^{15-18}$ the food industry, ${ }^{19-23}$ environmental analysis ${ }^{24-27}$ and biology ${ }^{28,29}$ ) with significant socio-economic impact. Importantly, LIBS has also been applied to the analysis of nuclear reactor materials. ${ }^{30,31}$ Even though LIBS is a well proven technique for the detection of trace elements at high and moderate concentrations, getting to lower and lower detection limits for all elements is an ongoing challenge for the LIBS community to meet. ${ }^{32-34}$

Different approaches have been adopted, in an endeavour to enhance the sensitivity of LIBS. In this context, e.g., the so-called

${ }^{a}$ Dublin City University, School of Physical Sciences and NCPST, Dublin 9, Ireland. E-mail: syedah.zehra2@mail.dcu.ie

${ }^{b}$ School of Physics, University College Dublin, Dublin, Ireland

'Università degli Studi di Padova, LUXOR CNR IFN, Italy

${ }^{d}$ National Research Council-Institute for Photonics and Nanotechnologies CNR IFN, Via Trasea 7, 35131 Padova, Italy dual pulse excitation scheme makes use of two laser beams in either a collinear or an orthogonal arrangement, to enhance the signal to background ratio (SBR) of the LIBS signal. ${ }^{18,30}$ Specifically, several LIBS studies investigating the optimization of limit of detection (LoD) of carbon in steel, have been reported ${ }^{35-42}$ including Vacuum-UV (VUV) LIBS that facilitates the detection of light elements. ${ }^{32,35,41}$

The Bessemer process heralded the era of modern steelmaking in the late 1850s. Since that time, steel has become crucial for the world's industrial economy due to its impact in the fields of: construction, automotive and ship building. The competitiveness of the steelmaking industry critically depends on the development of novel techniques for the production of steel with lower-impurity levels. One known such impurity is carbon, which determines the mechanical strength and hardness, making its machining more difficult. The key role of carbon in the mechanical properties of steel renders it critical for the steel industry. For example, low carbon steel (0.005$0.25 \mathrm{wt} \%$ ) possesses low strength and high ductility. Hence it has applications in e.g., automobile body components, pipes, concrete construction components and food cans. Medium carbon steel $(0.25-0.60 \mathrm{wt} \%)$ exhibits midlevel strength and ductility. Hence it is used in e.g., railway tracks, train wheels, crankshafts and gearboxes. High carbon steel (0.60-1.25 wt\%) is characterised by high strength combined with low ductility. Hence it is especially important where high wear-resistance and hardness are critical characteristics of a material. Examples include cutting tools, springs, high strength wire and dies. Thus 


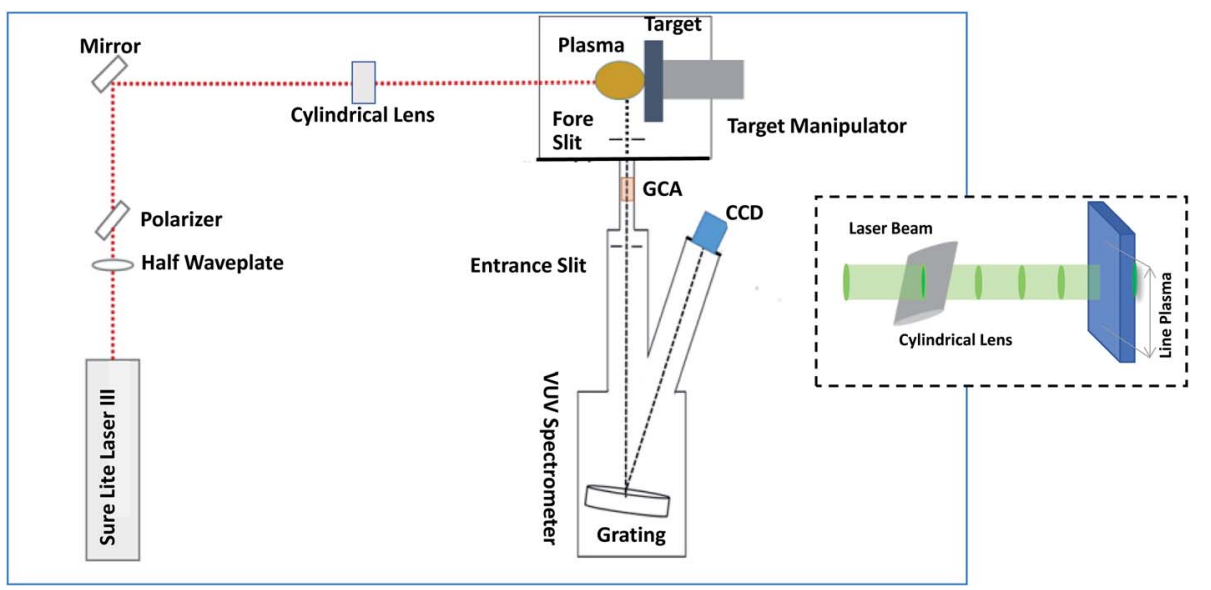

Fig. 1 Schematic diagram of the VUV LIBS experimental setup. A plano-cylindrical lens ( $\mathrm{f}-130 \mathrm{~mm}$ ) was used to focus the laser beam onto the target samples. An adjustable iris (not shown) was placed in front of the lens in order to adjust the length of the plasma column. The VUV emission spectra were obtained by a means of a $1 \mathrm{~m}$ normal incidence vacuum spectrometer.

the identification and accurate determination of carbon and its concentration in all steels is crucial.

Although in principle LIBS is a straightforward technique, there are a variety of experimental parameters that affect its performance. ${ }^{43-45}$ These pertain to the main components such as the laser pulse characteristics, the target shape, the sensitivity, resolution and type of spectrometer and also the type of detection.

Besides the above mentioned parameters, the focusing geometry plays an important role in LIBS measurements. In that case, a thorough understanding of the impact of parameters such as the lens type (e.g. cylindrical, sphere-cylindrical or spherical), the focal length of the lens, the distance between the lens and the sample, and the angle of laser incidence ${ }^{32}$ have on the experimental findings, is required.

Line focused plasmas, created by cylindrical lenses ${ }^{32,45,46}$ have been the subject of investigations for different applications. Rodolfa and Cremers ${ }^{47}$ used cylindrical optics to create a linear spark for spatial surface mapping whereas Mateo et al. ${ }^{46}$ used this geometry for fast atomic mapping of heterogeneous surfaces.

In this work, emphasis is placed on the effect of the focusing geometry on VUV LIBS for carbon in steel. Specifically, the key performance parameters for LIBS, ${ }^{32,48}$ namely signal to background ratio (SBR) and limit of detection (LoD) are compared for time integrated, space resolved (TI-SR) VUV LIBS for both point and line focus geometries. Experiments on the latter are performed, specifically the effect of focusing and the location of the plasma with respect to the optic axis of the spectrometer is explored in detail. Thus, the measurements presented here, consitute a systematic investigation aiming to highlight the potential enhancement of the performance of a VUV LIBS system by applying an apparently simple, but impactful, modification.

\section{Experimental setup}

A schematic of the experimental setup is shown in Fig. 1. The light source was an Nd:YAG Continuum Surelite III-10 laser, generating pulses of $6 \mathrm{~ns}$ duration at a central wavelength of $1064 \mathrm{~nm}$. The maximum pulse energy was $650 \mathrm{~mJ}$ at a repetition rate of $10 \mathrm{~Hz}$. The energy was controlled by a combination of a half waveplate and a polarizer. The laser beam was focused by a $130 \mathrm{~mm}$ focal length plano-convex cylindrical lens $(1064 \mathrm{~nm}$ antireflection coated). As a plano-cylindrical lens was used, focusing occurred in only one direction and the length of the line formed depended on the diameter of the laser beam. The length of plasma column so formed could then be adjusted by controlling the diameter of the laser beam. This was done by placing an aperture (an adjustable iris) in the beam before it was incident on the lens. The effect of defocusing and of the absolute target position with respect to the optic axis of the spectrometer were both important parameters to be varied and optimised to obtain the best possible LoD values.

The spectra were obtained with the aid of a $1 \mathrm{~m}$ normal incidence spectrometer (Acton Research Corporation VM521), operated in an off-Rowland circle arrangement and equipped with a 1200 grooves $\mathrm{mm}^{-1}$ grating $\left(\mathrm{Al} / \mathrm{MgF}_{2}\right.$ coated). The width of the entrance slit of the spectrometer was set to $50 \mu \mathrm{m}$. The registration of the spectra took place via a $2 \mathrm{D}$ Andor ${ }^{\mathrm{TM}}$ backilluminated charge-coupled device (CCD). The interaction chamber and the spectrometer were pumped down to a base pressure of $c a \cdot 10^{-6}$ mbar. A Glass Capillary Array (GCA) has been used to maintain differential pumping conditions between the target chamber and the spectrometer. ${ }^{32}$ The targets used here (tabulated in Table 1), were all standard, certified reference (carbon/steel) metals purchased from Glen Spectra Reference Materials ${ }^{\mathrm{TM}}$ presented to the laser as flat surfaces aligned with the optic axis of the spectrometer. Importantly the long axis of the plasma is aligned vertically, to lie along the direction of the entrance slit of the spectrometer.

This geometry leads to a significant enhancement in the signal level over that provided by a point-like plasma source, for a number of reasons. Firstly, the normal incidence spectrometer configuration is quite stigmatic. This, combined with fact that the line plasma can be considered to behave like a quasi 
Table 1 Steel samples and the respective carbon concentrations

\begin{tabular}{llc}
\hline No. & Certified Sample Label & Concentration $(\mathrm{ppm})$ \\
\hline 1 & CRM Fe 1/1 & 50 \\
2 & 11 CM 5078 & 180 \\
3 & 108 BS XAAS & 410 \\
4 & CRM 12 B 1767 & 520 \\
5 & IARM-206A 206A & 680 \\
6 & CRM 12X 15252 & 940
\end{tabular}

continuous array of point sources, means that VUV radiation from the full length of the vertical line plasma can be captured and imaged onto the CCD camera. Thus, when the CCD is operated in full vertical binning mode (i.e., when the full 512 pixels constituting each CCD column are summed vertically), an enhancement of the signal to noise ratio (SNR), compared to that yielded by the point plasma geometry, is observed. Alternatively, one can view the line plasma source as giving rise to a vertically stacked array of point like plasma spectra on the CCD, which are subsequently aggregated to yield a single one dimensional (1D) spectrum with decreased quantum noise.

Regarding the space resolved measurements, a $250 \mu \mathrm{m}$ wide fore slit, aligned vertically with the entrance slit of the spectrometer, was placed between the target and entrance slit of the spectrometer allowing for selection of specific slices of the expanding plasma plume. At the early stages of the plasma expansion, continuum emission, bremsstrahlung and recombination radiation dominate the observed spectrum. In addition, the emission lines originating from bound-to-bound transitions in the hot plasma core are strongly broadened by the Stark effect such that close lying lines are blended into a quasicontinuum close to the target surface. Thus, the key role of the foreslit is to minimize the contribution of continuum emission from the hot plasma core, thus improving the signal to background ratio. Overall the goal is to obtain a high SNR for the emission line along with a high SBR. The achievement of the former optimises the slope sensitivity ' $S$ ' for the concentration calibration curve while the latter ensures a low (relative ot the line) background count level. Since, for quantum noise with a Poisson distribution, the noise scales as the square root of the signal, the achievement of a low background ensures a low value for ' $\sigma$ ' the standard deviation and hence a low value for the limit-of-detection (LoD) which scale as $3 \sigma / S$.

\section{Effect of laser focus and target position}

The emission line at $97.7 \mathrm{~nm}$, corresponding to the $1 \mathrm{~s}^{2} 2 \mathrm{~s}^{2}{ }^{1} \mathrm{~S}_{0^{-}}$ $1 \mathrm{~s}^{2} 2 \mathrm{~s}^{1} 2 \mathrm{p}^{1}{ }^{1} \mathrm{P}_{1}$ transition in the $\mathrm{C}$ III spectrum was used to determine the LoD in this experiment. This line was selected due to its relatively high intensity with respect to the adjacent lines. The absence of any other strong emission lines in the spectral vicinity results in a low background level. Hence, it is the combination of high emission intensity (signal) along with low background, and low background fluctuation, amounting to a low standard deviation ' $\sigma$ ' for the background, that must be aimed for. In order to achieve such favourable conditions, the optimum position for both the cylindrical lens and the target should be established. Thus, a steel sample with a known carbon concentration of 520 ppm was used in experiments designed to determine the lens and target positions that optimised SNR, SBR and ultimately, LoD.

Measurements were performed for target positions (displacements from the optic axis of VM521 VUV spectrometer) ranging from $0.5 \mathrm{~mm}$ to $2.0 \mathrm{~mm}$, in steps of $0.5 \mathrm{~mm}$. These measurements were also performed at each target position for different lens focus and defocus positions, ranging from $12.5 \mathrm{~mm}$ to $7.5 \mathrm{~mm}$, in steps of $1 \mathrm{~mm}$, so that the optimum combination of the lens and target position is established.

Single shot spectra were captured, stored and aggregated after each experiment for post processing and analysis. For each of the spectra presented in this work, 25 such single shot spectra were recorded at each target and lens position and were then averaged. The signal to background ratio and the standard deviation of the background for the abovementioned set of target positions are plotted Fig. 2(a). The average background intensity was extracted by computing the integral of 11 pixels around $104.4 \mathrm{~mm}$, whereas the standard deviation of the
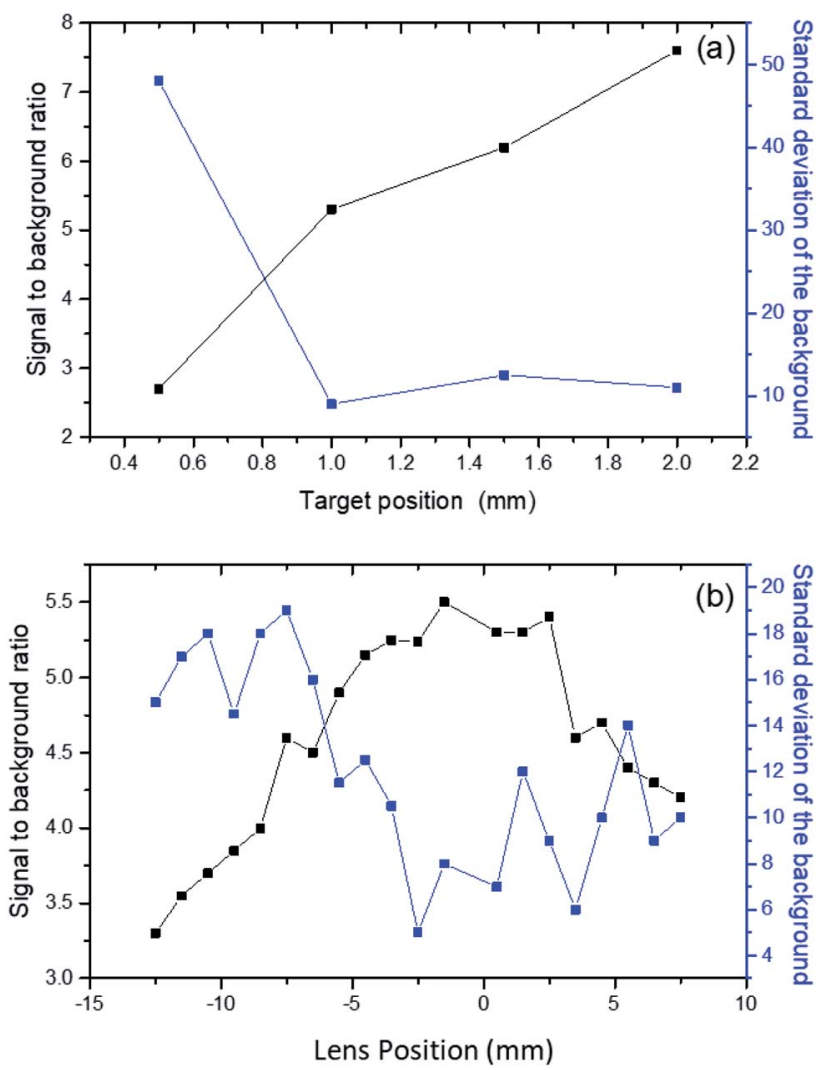

Fig. 2 Line $(97.7 \mathrm{~nm})$ and background (104.4 nm) intensities obtained (a) at four different target positions ranging from $0.5 \mathrm{~mm}$ to $2.0 \mathrm{~mm}$ and a fixed lens position (defocused by $+1.0 \mathrm{~mm}$ ) (b) at lens positions ranging from $-12.5 \mathrm{~mm}$ to $+7.5 \mathrm{~mm}$ at a fixed target position $(1.0 \mathrm{~mm})$. The signal to background ratio (SBR) and standard deviation of the background (SBR) at ca. $104.4 \mathrm{~nm}$ were extracted in both cases. 
background was calculated by averaging 150 spectra at every target and lens position as: $\sigma=\frac{\sqrt{\sum(x-300)^{2}}}{150}$, where 150 corresponds to the total number of accumulated spectra and $x$ represents the background level at a wavelength of $104.4 \mathrm{~nm}$ for each spectrum. As shown in Fig. 2(a), a target position of $1.0 \mathrm{~mm}$ yielded a low background signal, a low standard deviation of that background signal and a relatively high signal to background ratio (SBR) for the C III $97.7 \mathrm{~nm}$ line. Although a target position of $2.0 \mathrm{~mm}$ yielded a higher SBR, the $97.7 \mathrm{~nm}$ line signal was found to be less stable at that position and hence the $1.0 \mathrm{~mm}$ value was chosen for all subsequent experiments.

In a similar manner, the signal to background ratio and the standard deviation of the background for the set of lens positions mentioned above are plotted Fig. 2(b), for a fixed lens position of $1.0 \mathrm{~mm}$. As it is observed in this graph, the optimum lens position is around $2.5 \mathrm{~mm}$, above the target surface. This finding can be explained as follows: when the focal point is above the sample surface, the plasma temperature is expected to be higher because the plasma plume expands into the more tightly focused laser beam region where the laser beam power density is correspondingly higher than in the case where it is focused below the target surface. This behaviour has previously reported in studies involving point plasmas. ${ }^{32,49-51}$ Furthermore, the data presented in Fig. 2(b) yielded good SBR values for the $97.7 \mathrm{~nm}$ line, stable SBR values of $c a$. 5.5, along with low fluctuation in the background. For this reason, the target surface was located at a distance of $1 \mathrm{~mm}$ from the optic axis of the spectrometer and the lens focus was chosen to lie $2.5 \mathrm{~mm}$ in front of the target.

\section{Relative limit of detection (LoD) optimization}

Having established the optimum lens position and target position for the line plasma geometry, the next step is to determine the LoD for these optimum experimental conditions and compare it to measurements performed using the point plasma geometry. In addition, the present measurements are compared to previously reported LoD values obtained for point plasma focusing geometries, with the existing VUV LIBS setup, in order to highlight the more general trends in the performance of the system. It is noted that the performance of any LIBS system and the lowest achievable LoD value critically depends on experimental factors such as the source intensity, the spectrometer (VUV) transmission/throughput and the detector sensitivity/noise performance. Thus, the key objective of this section is to highlight the relative enhancement of the performance of this specific system, due to the modification of the focusing geometry.

\subsection{TI-SR VUV LIBS spectra for optimum SBR and LoD}

TI-SR VUV LIBS experiments were performed for line plasmas formed on six different steel targets of low carbon concentration (listed in Table 1). The data extracted from these spectra (shown

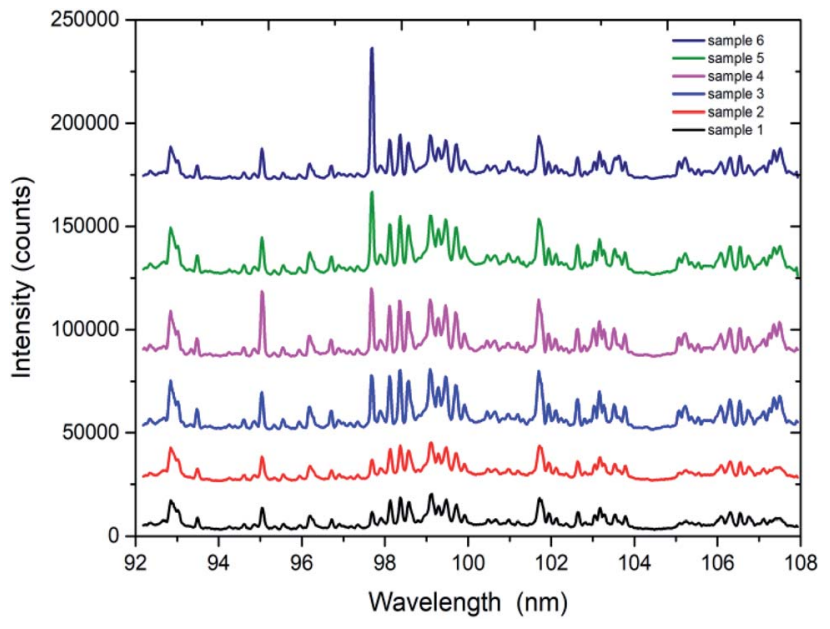

Fig. 3 Spectra (representing the average of 150 laser shots) obtained using samples 1 to 6 (Table 1) as reference target materials. Spectra are arranged in the order of concentration with the lowest carbon concentration as sample 1 and highest concentration level as sample 6 . The spectra are offset vertically in order to make them more visible to the reader.

in Fig. 3) were used to construct the calibration curve for the determination of the carbon content in unknown steel samples. The line intensity for each carbon concentration was obtained by taking the integral of 11 pixels around C III line at $97.7 \mathrm{~nm}$. These data were used to construct the calibration curve for carbon in steel and determine a limit of detection for the TI-SR VUV LIBS measurements performed using the line focus geometry.

\subsection{Calibration curve}

In order to construct the calibration curve, 150 single shot VUV spectra of each steel sample were averaged, from which the C III line at $97.7 \mathrm{~nm}$ was extracted and the background continuum level was determined. Background elimination for the emission line of interest was done by subtracting the integral of 11 pixels of the background signal (close to the $97.7 \mathrm{~nm}$ line at $104.4 \mathrm{~nm}$ ) from the integral of 11 pixels of the raw carbon emission line intensity values at $97.7 \mathrm{~nm}$. The background subtracted C III emission line intensity was then used to form the final calibration curve shown in Fig. 4 .

As a next step, the limit of detection and limit of quantification for carbon in steel were determined. In order to do so, the slope of the calibration curve ' $s$ ' and the variance in the background signal ' $\sigma$ ' had to be determined.

\subsection{Limit of detection (LoD) and limit of quantification (LoQ)}

On the basis of the calibration curve obtained in the previous section the limit of detection can be calculated. The formula used to obtain limit of detection (LoD) is:

$$
\mathrm{LoD}=\frac{3 \times \sigma}{s}
$$




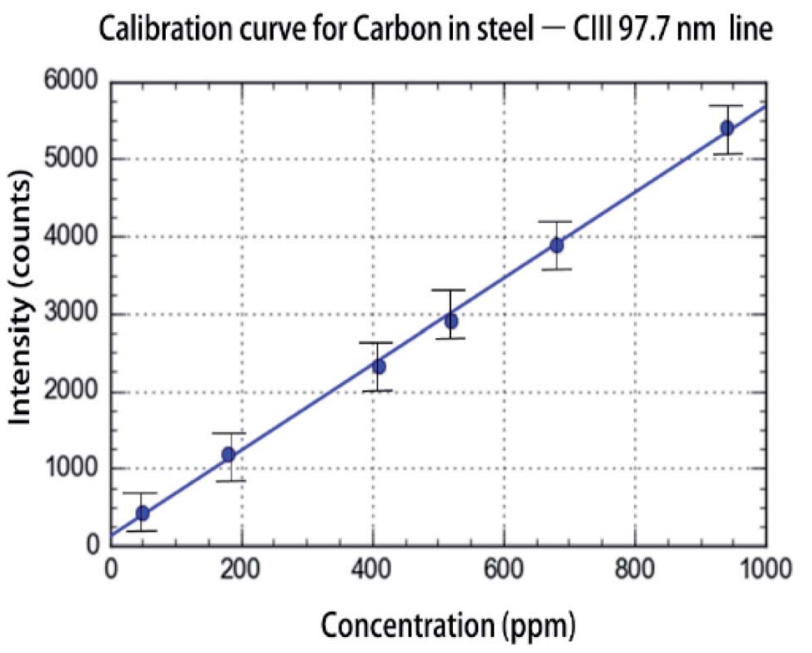

Fig. 4 The extracted calibration curve constructed using six different samples with carbon concentrations of 50 ppm to 940 ppm (cf. Table 1).

By definition it is the lowest quantity of a substance (analyte) which can be distinguished from the absence of that substance with some defined confidence level, typically $99.7 \%$, or $3 \sigma .^{52}$ The latter is the signal corresponding to the absence of the analyte, in this case carbon, is sometimes referred to as the limit of blank (LOB). The limit of detection may be obtained from the calibration curve (Fig. 4$)$ by dividing the slope $(s=5.6)$ of the calibration plot by three times the standard deviation of the background signal (93 counts) for the lowest concentration target. The lowest concentration target was used to determine the LoD for the worst-case scenario. Thus, the limit of detection is given as:

$$
\mathrm{LoD}=\frac{3 \times(\sigma=93)}{s=5.6}=\sim 50 \mathrm{ppm}
$$

For the case of TI-SR VUV LIBS of carbon in steel, using line plasma formation, the limit of detection is found to be $\sim 50 \mathrm{ppm}$ (or $50 \mu \mathrm{g} \mathrm{g}^{-1}$ ) as calculated above. The corresponding limit of quantification (LoQ) is usually taken as 3.3. $x \mathrm{LoD}^{50}$ and hence it is $167 \mathrm{ppm}$ ( or $167 \mu \mathrm{g} \mathrm{g}^{-1}$ ) in this work. These values are then compared with those obtained from VUV LIBS measurements made with point plasmas, tabulated in Table 2 . The target displacement values are selected according to the previously reported measurements performed with the existing VUV LIBS setup.

The comparison suggests that TI-SR VUV LIBS with a line plasma source improves the limit of detection for trace amounts of carbon in steel samples in the case of single pulse LIBS in vacuo. Specifically, if one introduces an 'improvement parameter' $\beta$ given as the ratio of the LoD corresponding to the point plasma geometry and that of the line plasma geometry:

$$
\beta=\frac{\text { LOD }_{\text {point plasma }}}{\text { LoD }_{\text {line plasma }}}
$$

Table 2 Comparison of LIBS parameters for point and line plasma geometries

\begin{tabular}{llllll}
\hline & Method (VUV) LIBS & $R^{2}$ & LoD (ppm) & SBR & $\beta$ \\
\hline 1 & TISR at 2 mm (point plasma) & 0.9880 & 224 & 2.4 & 4.5 \\
2 & TISR at 4 mm (point plasma) & 0.9998 & 316 & 2.8 & 6.3 \\
3 & TISR at 1 mm (line plasma) & 0.9998 & 50 & 4.4 & -
\end{tabular}

Up to a six-fold improvement in the value of beta can be achieved by using a line plasma focusing geometry, with a target surface displacement of $1 \mathrm{~mm}$. It can also be seen from Table 2 that a lower beta (4.5) is obtained for a target displacement of 2 $\mathrm{mm}$. Since the value of the SBR is greater in this case, the result looks rather counter intuitive. However, it can be seen that the $R^{2}$ value for the fit to the calibration curve is poorer than in the $4 \mathrm{~mm}$ case. This is due to a greater fluctuation in the data point spread and a lower value of the slope sensitivity $S$.

Even though this work is a comparative study between the two focusing geometries, the measurements obtained here should be compared to previously reported experiments performed with the existing VUV LIBS system, for the sake of completeness. The earliest measurement of the LoD for carbon in steel using the current TI-SR VUV LIBS setup was carried out by Khater et al. ${ }^{35}$ in 2002 while a later measurement was carried out by Xi and co-workers. ${ }^{32}$ Both investigators performed LIBS in single pulse mode by placing the steel target at $4 \mathrm{~mm}$ from the optic axis of the spectrometer. Xi et al. obtained a LoD of $54 \mathrm{ppm}$ in vacuo, while Khater and co-workers reported a value of $1.2 \mathrm{ppm}$ in air at very low pressure $0.1 \mathrm{mbar}$. The authors did not measure the LoD in vacuo but did mention a signal to background ratio that was 5 times lower in that case. Hence, one can expect the LoD at that time to lie somewhere between 5 and $10 \mathrm{ppm}$ for vacuum conditions which is at least 5 times lower than when Xi Jiang performed her experiments and obtained a LoD of $54 \mathrm{ppm}$. The latter experiment was repeated in this work and yielded a LoD value of $316 \mathrm{ppm}$ ( $c f$. Table 2). Thus, it is inferred that it is the lower performance of the LIBS optical system that should account for the deterioration of the absolute LoD values. Specifically, as mentioned above, experimental factors such as the source intensity, the spectrometer (VUV) transmission/throughput and the detector sensitivity/noise performance critically affect the performance of the system. As a result, a direct comparison with those previously reported measurements and LoD values reported by other groups (see e.g. ref. 36-41) is beyond the scope of this work.

\section{Conclusion}

This work reports LIBS measurements performed in time integrated-space resolved (TI-SR) mode, using a planocylindrical lens to ensure the formation of a line plasma. As with a point plasma there is a vast parameter space available to improve the LIBS performance. In this study the parameter investigated was the focusing geometry. Using this geometry, the optimum optical emission was obtained when the target 
was displaced by a distance of $1 \mathrm{~mm}$ from the optic axis of the spectrometer and the cylindrical lens was displaced by $2.5 \mathrm{~mm}$ so that its focus position lay $2.5 \mathrm{~mm}$ above the target. Furthermore, the calibration curve was constructed and the LoD for the TI-SR VUV LIBS was obtained for point and line plasma plumes in vacuo. These results were compared with measurements acquired using the point plasma geometry, showing a significant improvement of the limit of detection (LoD).

Regarding the absolute LoD value, it is not feasible to compare it with the previously reported values due to the deterioration of the performance of the existing LIBS optical system. Clearly, new spectrometer optics and CCD detection would improve the absolute value of the LoD, restoring it to the earlier values. In addition, the optimal use of background gases $^{35}$ along with the implementation of double pulse excitation $^{32}$ would significantly improve the value reported here. Future improvements will also include extension of the current option for single channel, time resolved VUV detection, ${ }^{53}$ to multichannel VUV detection which is well known to improve the LoD for UV-Visible LIBS. ${ }^{41}$

\section{Conflicts of interest}

There are no conflicts to declare.

\section{Acknowledgements}

SSZ acknowledges the award of an Education, Audio-visual and Culture Executive Agency (EACEA) fellowship from the Erasmus Mundus Joint Doctorate Programme EXTATIC, Project No. 2012-0033 This work is supported by the Sustainable Energy Authority of Ireland (SEAI) Grant No. 19/RDD/556 and Science Foundation Ireland (SFI) Grant No. 19FFP/6956 and 16/RI/3696. The work is associated with EU H2020 COST Action No. CA17126 (TUMIEE).

\section{References}

1 L. J. Radziemski, Spectrochim. Acta, Part B, 2002, 57, 11091113.

2 L. Radziemski and D. Cremers, Spectrochim. Acta, Part B, 2013, 87, 3-10.

3 A. De Giacomo and J. Hermann, J. Phys. D: Appl. Phys., 2017, 50, 183002.

4 P. K. Carroll and E. T. Kennedy, Contemp. Phys., 1981, 22, 6196.

5 E. T. Kennedy, Contemp. Phys., 1984, 25, 31-58.

6 J. L. Gottfried, F. C. De Lucia, C. A. Munson and A. W. Miziolek, Anal. Bioanal. Chem., 2009, 395, 283-300.

7 J. Moros, J. A. Lorenzo and J. J. Laserna, Anal. Bioanal. Chem., 2011, 400, 3353-3365.

8 V. Lazic, A. Palucci, S. Jovicevic and M. Carpanese, Spectrochim. Acta, Part B, 2011, 66, 644-655.

9 R. Gaudiuso R, M. Dell'Aglio M, O. De Pascale, G. S. Senesi and A. De Giacomo, Sensors, 201, 10, 7434-7468.

10 A. Giakoumaki, K. Melessanaki and D. Anglos, Anal. Bioanal. Chem., 2007, 387, 749-760.
11 P. Pouli, K. Melessanaki, A. Giakoumaki, V. Argyropoulos and D. Anglos, Spectrochim. Acta, Part B., 2005, 60, 11631171.

12 F. Ruan, T. Zhang and H. Li, Appl. Spectrosc. Rev., 2019, 54, 573-601.

13 K. Melessanaki, M. Mateo, S. C. Ferrence, P. P. Betancourt and D. Anglos, Appl. Surf. Sci., 2002, 197-198, 156-163.

14 A. Botto, B. Campanella, S. Legnaioli, M. Lezzerini, G. Lorenzetti, S. Pagnotta, F. Poggialini and V. Palleschi, J. Anal. At. Spectrom., 2019, 34, 81-103.

15 R. Noll, H. Bette, A. Brysch, M. Kraushaar, I. Mönch, L. Peter and V. Sturm, Spectrochim. Acta, Part B, 2001, 56, 637-649.

16 D. Bulajic, G. Cristoforetti, M. Corsi, M. Hidalgo, S. Legnaioli, V. Palleschi, A. Salvetti, E. Tognoni, S. Green, D. Bates, A. Steiger, J. Fonseca, J. Martins, J. McKay, B. Tozer, D. Wells, R. Wells and M. A. Harith, Spectrochim. Acta, Part B, 2002, 57, 1181-1192.

17 L. Sun, H. Yu, Z. Cong, H. Lu, B. Cao, P. Zeng, W. Dong and Y. Li, Spectrochim. Acta, Part B, 2018, 142, 29-36.

18 L. Sun, H. Yu, Z. Cong, Y. Xin, Y. Li and L. Qi, Spectrochim. Acta, Part B, 2015, 112, 40-48.

19 V. Juvé, R. Portelli, M. Boueri, M. Baudelet and J. Yu, Spectrochim. Acta, Part B, 2008, 63, 1047-1053.

20 W. Lei, V. Motto-Ros, M. Boueri, Q. Ma, D. Zhang, L. Zheng, H. Zeng and J. Yu, Spectrochim. Acta, Part B, 2009, 64, 891898.

21 H. Zhang, S. Wang, D. Li, Y. Zhang, J. Hu and L. Wang, Sensors, 2019, 19, 4225.

22 M. Markiewicz-Keszycka, X. Cama-Moncunill, M. P. CasadoGavalda, Y. Dixit, R. Cama-Moncunill, P. J. Cullen and C. Sullivan, Trends Food Sci. Technol., 2017, 65, 80-93.

23 S. Moncayo, S. Manzoor, J. D. Rosales, J. Anzano and J. O. Caceres, Food Chem., 2017, 232, 322-328.

24 R. Wainner, R. Harmon, A. Miziolek, K. McNesby and P. French, Spectrochim. Acta, Part B, 2001, 56, 777-793.

25 G. NicoLoDelli, G. S. Senesi, I. L. de Oliveira Perazzoli, B. S. Marangoni, V. De Melo Benites and D. M. B. P. Milori, Sci. Total Environ., 2016, 565, 1116-1123. 26 P. R. Villas-Boas, R. A. Romano, M. A. de Menezes Franco, E. C. Ferreira, E. J. Ferreira, S. Crestana and D. M. B. P. Milori, Geoderma, 2016, 263, 195-202.

27 R. S. Harmon, R. E. Russo and R. R. Hark, Spectrochim. Acta, Part B, 2013, 87, 11-26.

28 L. Sancey, V. Motto-Ros, B. Busser, S. Kotb, J. M. Benoit, A. Piednoir, F. Lux, O. Tillement, G. Panczer and J. Yu, Sci. Rep., 2014, 4, 6065.

29 Y. Gimenez, B. Busser, F. Trichard, A. Kulesza, J. M. Laurent, V. Zaun, F. Lux, J. M. Benoit, G. Panczer, P. Dugourd, O. Tillement, F. Pelascini, L. Sancey and V. Motto-Ros, Sci. Rep., 2016, 6, 29936.

30 E. J. Kautz, A. Devaraj, D. J. Senor and S. S. Harilal, Opt. Express, 2021, 29, 4936.

31 A. M. Roldán, M. Pisarčík, M. Veis, M. Držík and P. Veis, Spectrochim. Acta, Part B, 2021, 177, 106055.

32 X. Jiang, P. Hayden, J. T. Costello and E. T. Kennedy, Spectrochim. Acta, Part B, 2014, 101, 106-113. 
33 V. Sturm, B. Erben, R. Fleige and W. Wirz, Opt. Express, 2019, 27, 36855.

34 R. Noll, Laser-Induced Breakdown Spectroscopy, in LaserInduced Break. Spectrosc., Springer Berlin Heidelberg, Berlin, Heidelberg, 2012, pp. 7-15.

35 M. A. Khater, J. T. Costello and E. T. Kennedy, Appl. Spectrosc., 2002, 56, 970-983.

36 J. A. Aguilera, C. Aragón and J. Campos, Appl. Spectrosc., 1992, 46, 1382-1387.

37 C. Aragón, J. A. Aguilera and J. Campos, Appl. Spectrosc., 1993, 47, 606-608.

38 C. Aragón, J. A. Aguilera and F. Peñalba, Appl. Spectrosc., 1999, 53, 1259-1267.

39 L. Peter, R. Noll and V. Sturm, Appl. Spectrosc., 2000, 54, 1275-1278.

40 L. Peter, V. Sturm and R. Noll, Appl. Opt., 2003, 42, 6199.

41 V. Sturm, L. Peter and R. Noll, Appl. Spectrosc., 2000, 54, 1275-1278.

42 M. Hemmerlin, R. Meilland, H. Falk, P. Wintjens and L. Paulard, Spectrochim. Acta, Part B, 2001, 56, 661-669.
43 D. W. Hahn and N. Omenetto, Appl. Spectrosc., 2010, 64, 335A-336A.

44 D. W. Hahn and N. Omenetto, Appl. Spectrosc., 2012, 66, 347419.

45 A. S. Eppler, D. A. Cremers, D. D. Hickmott, M. J. Ferris and A. C. Koskelo, Appl. Spectrosc., 1996, 50, 1175-1181.

46 M. P. Mateo, S. Palanco, J. M. Vadillo and J. J. Laserna, Appl. Spectrosc., 2000, 54, 1429-1434.

47 K. T. Rodolfa and D. A. Cremers, Appl. Spectrosc., 2004, 58, 367-375.

48 M. A. Khater, J. T. Costello and E. T. Kennedy, Appl. Spectrosc., 2002, 56, 970-983.

49 S. S. Harilal, R. W. Coons, P. Hough and A. Hassanein, Appl. Phys. Lett., 2009, 95, 221501.

50 S. S. Harilal, P. K. Diwakar and A. Hassanein, Appl. Phys. Lett., 2013, 103, 41102.

51 F. J. Fortes, L. M. Cabalín and J. J. Laserna, Spectrochim. Acta, Part B, 2008, 63, 1191-1197.

52 A. Shrivastava and V. Gupta, Chron. Young Sci., 2011, 2, 21.

53 S. S. Zehra, J. T. Costello, P. Nicolosi and P. Hayden, Proc. SPIE, 2018, 10674, 106741H. 